\title{
TRIBUTACIÓN DEL SECTOR MINERO Y RECUPERACIÓN DEL MEDIO AMBIENTE EN PERÚ, 2011-2016
}

\author{
THE MINING SECTOR TAXATION AND THE ENVIRONMENT RECOVERY IN PERU, \\ 2011-2016
}

Adolfo ValenCIA GutIÉRREZ
Universidad Nacional Mayor de San Marcos
Lima, Perú
ORCID: https://orcid.org/0000-0002-3757-8071
Correo electrónico: avalenciag@unmsm.edu.pe

[Recibido: 18/02/2020 Aceptado: 30/07/2020 Publicado: 28/12/2020]

\section{RESUMEN}

Objetivo: Determinar si el monto de los tributos de las empresas formales del sector minería peruana compensa los daños al medio ambiente o ecosistemas ocasionados a razón de sus actividades. Método: Investigación de enfoque cuantitativo deductivo, no experimental y transversal, de nivel explicativo - causal. La población objetivo estuvo conformada por 49 empresas mineras formales que generan tributos al Estado peruano en 15 regiones, evidenciado con documentos normativos del Ministerio del Ambiente. Resultados: Los ingresos tributarios del sector minero no han sido utilizados para la conservación y restauración del medio ambiente destruido por la explotación minera; asimismo, la gestión del Ministerio del ambiente ha sido ineficiente por lo que se concreta la necesidad de una tributación medio ambiental. Conclusiones: Se corroboró que la tributación del sector minero ha sido parte de los ingresos del Estado peruano, sin embargo, no se pudo determinar si compensa o no los daños causados al medio ambiente, ya que el Estado no ha elaborado, formulado ni ejecutado proyecto alguno en relación con la conservación y restauración del medio ambiente.

Palabras clave: Ambiente; minería; tributación ambiental; tributación.

\begin{abstract}
Objective: Determine if the taxes amount formal companies in the Peruvian mining sector compensates the environment damage or ecosystems caused by their activities. Method: Research of deductive, non-experimental and transversal quantitative approach, explanatory - causal level. The target population consisted of 49 formal mining companies that generate taxes on the Peruvian State in 15 regions, evidenced with normative documents from the Ministry of the Environment. Results: The mining sector tax revenues for the conservation and restoration of the destroyed environment by mining; likewise, the Ministry of the environment management has been inefficient so it develops the need for an environmental taxation. Conclusions: It corroborated that the taxation of the mining sector has been part of the Peruvian state income; however, it could not be determined whether or not it compensates to the environment damage, since the state has not elaborated, formulated or implemented any project in relation to the conservation and restoration of the environment.
\end{abstract}

Keywords: Environment; mining; environmental taxation; taxation.

(c) Los autores. Este artículo es publicado por la Revista Quipukamayoc, Universidad Nacional Mayor de San Marcos. Este es un artículo de acceso abierto, distribuido bajo los términos de la Licencia Creative Commons Atribución-NoComercial-Compartirlgual 4.0 Internacional.(http://creativecommons.org/licenses/by-nc-sa/4.0/), que permite el uso no comercial, distribución y reproducción en cualquier medio, siempre que la obra original sea debidamente citadas. 


\section{INTRODUCCIÓN}

El desarrollo de la investigación tiene su punto de partida en la verificación y constatación in situ de la realidad en cuanto se refiere a los perjuicios perpetrados en el ambiente debido a las actividades extractivas mineras formales en el Perú y su relación con la tributación compensatoria de este sector. Se suma a ello la inversión y el gasto público destinado por el Estado peruano para efectos de conservación y restauración de las afectaciones en el medio ambiente. Se tiene en consideración tres aspectos importantes: el primero se vincula con los daños perpetrados en los ecosistemas, debido a las actividades mineras formales; el segundo, con los volúmenes de tributación generados por el sector minero; y el tercero responde a la acción o inacción del aparato estatal para fines de compensación o reparación de los daños perpetrados.

Según el Observatorio de Conflictos Mineros en América Latina, OCMAL (2018) en el Perú se han producido 39 conflictos con relación a los efectos negativos al medio ambiente. Los principales conflictos se presentan a continuación:

El Proyecto La Morada pertenece a la empresa minera Miski Mayo. Esta tiene su ubicación en la región Cajamarca, jurisdicción de los distritos Pedro Gálvez, en la provincia de San Marcos; Cachachi, en la provincia de Cajabamba; y el de Jesús, en la provincia de Cajamarca. Desde el inicio de las operaciones en el 2004, se han suscitado diversos hechos que evidenciaron un conjunto de malas prácticas y políticas empresariales implementadas por los funcionarios de la transnacional brasilera que generó el descontento de los pobladores, quienes tuvieron que organizarse con la finalidad de expulsar a la empresa de sus propiedades. Esto provocó que la empresa organice grupos armados para repeler a los pobladores, lo que originó el conflicto social.

El Proyecto Río Blanco pertenece a la empresa Monterrico Metals. Río Blanco Copper S.A. es un yacimiento de cobre ubicado en el lugar denominado Henrys Hill, comunidad de Segunda y Cajas, región Piura. Debido a las alertas y falta de comunicación referente al impacto ambiental que podría generar la actividad minera en el agua y ecosistemas, las comunidades reaccionaron en contra del proyecto ya que este se circunscribe a los bosques neblinosos, que son parecidos a una esponja porque sirven para capturar, filtrar y distribuir el agua de la que depende la costa desértica piurana. Los impactos del proyecto minero en el medio ambiente, principalmente en el agua y consecuentemente en el desarrollo del agro, se configuraban significativos y los conflictos se agudizaron a partir del 2003 con resultados fatales: dos muertos y varios heridos.
El Proyecto minero La Zanja es un yacimiento de oro y plata de las empresas Compañía de Minas Buenaventura, Newmont Mining Corporation y Minera la Zanja SRL, situado en jurisdicción del Caserío La Zanja, provincia Santa Cruz, región de Cajamarca, comprende dos unidades mineras: yacimientos San Pedro y Pampa Verde, cuya explotación sería a tajo abierto, cuyo efecto sería un material estéril del orden 5,9 millones de toneladas. Desde el inicio de sus actividades en el 2004, los pobladores de Santa Cruz y San Miguel se manifestaron opuestos en razón de la contaminación del agua, por el uso de lixiviación, y por estar ubicado en una cabecera de cuenca, afectando provincias de Cajamarca y Lambayeque. La oposición de las rondas campesinas y el Frente de Defensa de Santa Cruz obligó a la empresa a postergar el proyecto. En el 2007, los pobladores de Santa Cruz hicieron un paro de dos semanas demandando el retiro de la empresa Buenaventura. Denunciaron la muerte de 5 mil truchas en el río Pisit por posible derrame de insumos químicos de las pozas del proyecto La Zanja; hecho reportado por la ONG Ecovida, que identificó la causa principal en las altas concentraciones de plomo. La empresa anunció que el proyecto está en marcha y que espera el Estudio de Impacto Ambiental correspondiente.

El Proyecto minero Yanacocha es un yacimiento de oro, a cargo de la empresa Newmont Mining Corporation, con Estudio de Impacto Ambiental (EIA) aprobado. Está ubicado en Cerro Quilish, región de Cajamarca; inició la explotación en 1993, con una reserva de 333 mil onzas de oro. Después de la exploración, desde 1995 al 2000, declara la existencia de reservas probables de 3700000 de onzas de oro recuperable, adicionalmente 500000 onzas potencialmente recuperables. La empresa secó el río Yanacocha, el cual es un ecosistema frágil que incluye la presencia de microcuencas que abastecen a los ríos grandes Poecón y Quilish, siendo el 70\% del agua que abastece a Cajamarca. El inicio de las operaciones fue altamente conflictivo; las poblaciones a tomaron acciones legales contra la empresa, que ganó en Lima en el 2004, lo que provocó una oleada de protestas que llevaron a un paro masivo del 2 al 16 de setiembre del 2004. El caso debe ser tomado como un ejemplo de conflicto en torno al medio ambiente originado por las operaciones mineras sin las garantías sociales, por la falta de un Estado comprometido con la preservación del medio ambiente y por un Poder Judicial de espaldas a la realidad.

El Proyecto minero Conga, de oro y otros metales, de la minera Yanacocha, está ubicado en la circunscripción de las provincias de Cajamarca y Celendín en sus cuencas hidrográficas. De acuerdo con el estudio de impacto ambiental del proyecto, este comprende dos tajos abiertos, uno de $2 \mathrm{~km}$ y otro de $1 \mathrm{~km}$ de profundidad, emplazados sobre la laguna El Perol. Removería diariamente 92000 
toneladas de roca en un lapso de 17 años, lo que generaría un promedio de 85000 toneladas de relaves y desechos tóxicos ubicados en un área de 700 ha próxima a la Laguna azul (total 40 lagunas amenazadas) y a los bofedales (100 hectáreas amenazadas) que dan vida al río Jadibamba, destinado a la agricultura, ganadería y consumo humano del distrito de Huasmín. En primer lugar, según Mirella Gallardo, ingeniera agrícola especialista en recursos hídricos y temas ambientales, exasesora técnica del PDRS-GTZ (hoy GIZ), el conflicto provocado por el proyecto minero Conga resulta de un derecho de la población a ser sujetos de consulta, así como de ser partícipe de los beneficios que genere la inversión minera. Asimismo, la inviabilidad del proyecto es refrendada por el científico Robert Moran, quien lo califica como un documento de mala elaboración y de escasa honestidad, debido a que no proporciona la información de nivel técnico suficiente, de modo que tanto población como organismos de regulación asuman decisiones en cuanto a control y fiscalización. Por ende, se califica a este documento de estar orientado solo a apelar a las relaciones públicas, facilitar los permisos y una total ausencia de un enfoque económico en perjuicio de las poblaciones afectadas.

El Proyecto minero Pierina, mina de la empresa canadiense Barrick Goldes de tajo abierto, está situada en la circunscripción del distrito de Jangas, de la provincia de Huaraz, en la región Áncash. Diariamente remueve 80 000 toneladas métricas entre minerales y desmonte, en su proceso de obtención del oro y la plata se recurre a una solución de lixiviante de cianuro de sodio como parte de un proceso cerrado que no produce relaves. Los engaños, contaminación y destrucción han provocado la disconformidad de la población, autoridades regionales y locales contra la empresa, por el no pago de los impuestos adeudados al Estado, por un monto de 140 millones de dólares, así como por la contaminación del medio ambiente, especialmente los recursos hídricos, y por la represión de la empresa contra la población que dejó dos víctimas fatales.

El Proyecto minero Antamina, de la Minera Antamina S. A. que tiene como accionistas a cuatro empresas líderes en la minería mundial: STRATA con el 33,75\%, BH BILLITON PLC con el 33,75 \%, TECK con el 2,5\% y MITSUBISHI CORPORATION con el 10\%, está ubicado en la comunidad de Juproq, región Áncash. Su explotación es de tajo abierto (con aproximadamente $2000 \mathrm{~m}$ de largo, $1000 \mathrm{~m}$ de ancho y $500 \mathrm{~m}$ de profundidad), remueve 360 000 toneladas diarias de material para un proceso de obtención de productos como: molibdeno, cobre, zinc, plata y plomo. Los conflictos con la comunidad de Juprog se vienen dando a causa de los límites con los terrenos de la mina y el avance de su explotación. La comunidad, ente otras peticiones, exigió la realización de un estudio de impacto en la salud de los comuneros a cargo del Centro de Salud Ocupacional y Protección del Ambiente para la Salud (CENSOPAS). Los resultados demostraron un alto riesgo para la salud de los comuneros a causa de la exposición a metales pesados, por lo cual, quedó comprobado la presencia de estos metales en la sangre por encima de los límites permisibles.

El Proyecto La Oroya, de las empresas Volcan Cía. S. A. A. y Doe Run Perú, tiene a su cargo la fundición ubicada en la provincia de la Oroya, región Junín. Esta ha venido generando graves daños al entorno ambiental desde el año 1922 hasta la fecha, con la emisión de dióxido de azufre y sobre todo metales pesados, como el plomo, que son dañinos para la salud de los pobladores. La Oroya no solamente funde los minerales polimetálicos procedentes de la mina de Cerro de Pasco, además, procesa y funde otros minerales que por razones ambientales no se realiza en otros países. A causa de ello, los niveles de plomo en la sangre, especialmente en los niños, supera los índices máximos determinados por la OMS. La ciudad de La Oroya también figura como una de las poblaciones más contaminadas del planeta. Los niveles de contaminación provocados por la fundición han obligado a tomar acciones a las organizaciones ambientales peruanas, formándose así una mesa técnica que plantee soluciones al problema priorizando el apoyo a las acciones de la comunidad en defensa de sus derechos fundamentales.

De acuerdo con la Sociedad Nacional de Minería, Petróleo y Energía-SNMPE (2018), en cuanto se refiere a la tributación del sector minero y a la recuperación del medio ambiente, se ha considerado en la investigación, partiendo del hecho y realidad de la tributación formal o legal del sector minero, los aportes tributarios de las 49 empresas mineras constituidas legalmente según las leyes peruanas, considerando asimismo a este sector como uno de los principales contribuyentes al Estado peruano.

Así se determina las sumas de la tributación para el periodo comprendido entre el año 2011 y el año 2016, de acuerdo con la información de la Superintendencia Nacional de Aduanas y de Administración Tributaria - SUNAT (SUNAT, 2016).

Conocidos los daños a los ecosistemas causados por la explotación minera, considerando los datos consignados por el Observatorio de Conflictos Mineros en América Latina - OCMAL, que consigna 39 conflictos mineros latentes en el Perú, se plantea el problema de la investigación en términos del gasto de inversión del Estado en la restauración de los daños, teniendo como referencia la tributación del sector minero. Si bien estos tributos acordes a ley se integran en el ingreso total recaudado y son la base del gasto público conforme el presupuesto del Estado, el problema también se plantea en términos de la política del Estado 
en su prioridad por la conservación del medio ambiente, en la investigación de los gastos del Estado para restaurar los daños causados a los ecosistemas que permanecen alterados por la explotación minera. Así, la investigación, sobre esta situación problemática, formula los problemas de la investigación y basa su propuesta de la creación de una Tributación Ambiental como una de las alternativas.

Pizarro (2016) sostiene que el afán desmesurado de lucro de las compañías mineras no considera "los daños al ser humano, los animales, a la biósfera, etc.; pues la minería es, por lo general, responsable de daños irreparables al medio ambiente, los cuales traen como resultado el acelerado calentamiento global en el mundo" (p. 43).

Aquí es importante remarcar lo que el estudioso Rafael Tobia Díaz señala respecto de la tendencia doctrinal de conceptualizar a los "tributos verdes" como instrumentos de política económica ambiental o de gestión ambiental, para consolidarse como auténticos tributos que sirvan para la recaudación vinculada a la protección del medio ambiente (Tobia, 2019).

Asimismo, la Asociación Interamericana para la Defensa del Ambiente - AIDA (2014) considera, entre los más significativos impactos negativos al ambiente, la alteración y degradación penetrante y continua de cuerpos de agua, daños a la biodiversidad, la contaminación del aire por polvo, plomo y mercurio con subsecuentes problemas de salud de la población. Los grandilocuentes beneficios de corto plazo de la industria minera se reparten con desigualdad e insuficiencia. Por consiguiente, es vital una evaluación integral, detallada, técnica, transparente y con participación oportuna, efectiva y significativa de los actores de las comunidades para la toma de decisiones respecto del grado de viabilidad de determinado y evitar, de ese modo, los conflictos sociales.

Ante este panorama de conflictos sociales por los daños perpetrados por las empresas mineras en ecosistemas y que se ve reflejado, además, en la afectación directa a su población, el Estado ha creado una serie de mecanismos en virtud de le Ley General del Ambiente; sin embargo, poco o casi nada han hecho respecto a la defensa del ambiente y de los peruanos afectados. El Organismo de Evaluación y Fiscalización Ambiental - OEFA, como bien reza su lema, ejerce su fiscalización, pero facilitando la inversión en lugar de construir la confianza. Mediante Decreto Supremo No 001-2010-MINAM se inició el proceso de transferencia de las funciones de supervisión, fiscalización y sanción en materia ambiental del OSINERGMIN al OEFA. Sin embargo, Hugo Gómez Apac, expresidente del Consejo Directivo del OEFA, refirió que el organismo no tiene ningún afán de buscar procesos sancionadores y afirmó que las multas nunca han sido excesivas y ni siquiera cercanas al tope. Tal es así, como se revela en sus informes y lo señala el expresidente de su consejo directivo: "Desde que se creó en el 2008 se han realizado 5517 supervisiones a empresas, y en ese mismo periodo han derivado en multas firmes solo 96, el 1.7\%, es bajísimo. Eso significa que de cada 100 supervisiones que hace el OEFA menos de 2 terminan en un proceso sancionador que termina en una multa" (Mariluz, 2014). Las medidas correctivas de restauración y compensación ambiental que señalan las normativas legales peruanas parecen ser letra muerta.

\section{MATERIALES Y MÉTODOS}

La investigación desarrollada se encuadró dentro del enfoque cuantitativo deductivo. Se sustentó en un exhaustivo y pertinente marco teórico que fundamenta los lineamientos de las variables de estudio. El problema de investigación derivó en el objetivo para determinar si el monto de los tributos de las empresas del sector minería peruana compensa los daños al medio ambiente o ecosistemas ocasionados a razón de sus actividades; asimismo permitió el planteamiento de la hipótesis de que la tributación de las empresas mineras peruanas, en virtud a la carencia de implementación de una política ambiental por el Estado peruano, por el modo de integrarse y cómo logran distribuir las contribuciones, además debido a la falta de una objetiva valorización de los perjuicios perpetrados tanto al ambiente como a la sociedad en conjunto, no logran compensar los efectos dañinos ocasionados por sus actividades. Objetivo e hipótesis que han sido respondidas a la luz de resultados evidenciados por el procesamiento y el análisis de la data recogida haciendo uso de técnicas e instrumentos validados, para luego concretar en conclusiones y recomendaciones.

El estudio fue de tipo no experimental, ya que su proceso no exigió la manipulación deliberada de las variables de estudio ni la alteración de su objeto, sino que se observó los fenómenos en su contexto natural. Es transversal porque se hizo un corte en el tiempo, que corresponde al periodo entre los años 2011 y 2016, para conocer el problema. El alcance de la investigación fue de nivel explicativo-causal, por el carácter de las hipótesis y las estructuras de sus variables independientes, dependientes e intervinientes.

La investigación identificó y estableció las causas de la problemática del objeto expuesta en las hipótesis. Por características propias de la investigación y de acuerdo con el enfoque metodológico cuantitativo deductivo, el método apropiado y adoptado para el proceso de manejo de la data del estudio es el método estadístico. Bajo esta metodología se manejó una población 49 empresas de actividad minera formal que generan tributos al Estado peruano, 15 regiones afectadas por la actividad minera y 
un conjunto de documentos normativos del Estado peruano, pertenecientes al Ministerio del Ambiente en cuanto a presupuesto y gasto generado para conservar y restaurar el medio ambiente afectado. Los instrumentos de investigación observacional sirvieron para el recojo de datos útiles, el recuento, presentación, síntesis y el análisis que permitió arribar a conclusiones.

\section{RESULTADOS}

La tributación, como medio de control a la contaminación ambiental causada por la actividad minera, se sustenta en el hecho de que uno de los sectores económicos que más contamina el medio ambiente es el minero; por ello, la creación de tributos ambientales resulta un mecanismo efectivo de control para remediar la contaminación ambiental.

La tabla 1 muestra el volumen de los aportes tributarios del sector minería al fisco peruano, en el periodo 2011-2016, que sirvieron de parámetro para determinar, en caso de plantearse proyectos de conservación y restauración del medio ambiente, si compensa o no los daños producidos en el medio ambiente.

Tabla 1

Tributación del sector de minería en el Perú, 2011 - 2016 (en millones de soles)

\begin{tabular}{ccccc}
\hline $\mathbf{N}^{\circ}$ & AÑO & $\begin{array}{c}\text { MONTO TRIBUTACIÓN SECTOR } \\
\text { MINERO }\end{array}$ & $\begin{array}{c}\text { MONTO TRIBUTACIÓN TOTAL EN } \\
\text { PERÚ }\end{array}$ & $\begin{array}{c}\text { MONTO TRIBUTACIÓN SECTOR } \\
\text { MINERO EN \% DEL TOTAL }\end{array}$ \\
\hline 1 & 2011 & $15,152.7$ & $64,205.6$ & $23,6 \%$ \\
2 & 2012 & $15,077.9$ & $72,463.2$ & $20,8 \%$ \\
3 & 2013 & $11,549.9$ & $76,683.0$ & $15,06 \%$ \\
4 & 2014 & $11,824.2$ & $81,103,6$ & $14,6 \%$ \\
5 & 2015 & $6,865.7$ & $77,270.5$ & $8,9 \%$ \\
6 & 2016 & $6,012.0$ & $80,347.0$ & $7,5 \%$ \\
\hline \multicolumn{2}{c}{ TOTAL } & $66,482.4$ & $452,072.0$ & $14,7 \%$ \\
\hline
\end{tabular}

Fuente: Adaptado de los informes de la SUNAT, 2016 (http://www.sunat.gob.pe/)

Tabla 2

Proyectos elaborados y ejecutados por el MINAM para conservar y restaurar el medio ambiente afectado por explotación de minería.

\begin{tabular}{|c|c|c|c|c|}
\hline $\mathbf{N}^{\circ}$ & AÑO & PROYECTOS DEL MINAM & $\begin{array}{l}\text { PROYECTOS DEL MINAM - } \\
\text { CONSERVACIÓN RESTAURACIÓN } \\
\text { MEDIO AMBIENTE }\end{array}$ & $\begin{array}{l}\text { PROYECTOS EJECUTADOS DEL } \\
\text { MINAM - RESTAURACIÓN MEDIO } \\
\text { AMBIENTE }\end{array}$ \\
\hline 1 & 2011 & $\begin{array}{c}\text { 4.Códigos SNIP* } \\
129843 \\
63450 \\
141451 \\
171291\end{array}$ & 0 & 0 \\
\hline 2 & 2012 & $\begin{array}{l}\text { 4.Códigos SNIP* } \\
129843 \\
63450 \\
141451 \\
171291\end{array}$ & 0 & 0 \\
\hline 3 & 2013 & $\begin{array}{c}\text { 3.Códigos SNIP* } \\
129843 \\
141451 \\
1\end{array}$ & 0 & 0 \\
\hline 4 & 2014 & $\begin{array}{l}\text { 2.Códigos SNIP* } \\
129843 \\
141451\end{array}$ & 0 & 0 \\
\hline 5 & 2015 & $\begin{array}{c}\text { 1.Códigos SNIP* } \\
129843\end{array}$ & 0 & 0 \\
\hline 6 & 2016 & $\begin{array}{c}\text { 1.Códigos SNIP* } \\
129843\end{array}$ & 0 & 0 \\
\hline & TOTAL & 5 & 0 & 0 \\
\hline
\end{tabular}

Fuente: Adaptado de Portal de Transparencia, MINAM, 2016 (https://www.gob.pe/minam) ${ }^{\star}$ El 24 de febrero de 2017, quedó sin efecto el SNIP 
La tabla 2 muestra la ausencia de gestión del Estado peruano, por medio del Ministerio del Ambiente, con respecto a conservar y restaurar el medio ambiente afectado: ningún proyecto planteado ni ejecutado.

En la tabla 3 se corrobora la ausencia de gestión del Estado peruano cuando se observa que el presupuesto del Ministerio del Ambiente destinado para conservar y restaurar el medioambiente alterado y dañado por la actividad minera es cero, es decir, una total inacción.
En la tabla 4 se observa la misma situación con respecto al Presupuesto Institucional Modificado (PIM) del Estado y regiones afectadas en su medio ambiente por el sector minero y lo destinado para conservar y restaurar el medio ambiente afectado por la explotación de minería: cero, una inacción total por parte del Estado y las Regiones.

En la tabla 5 se aprecia que los montos recaudados tanto por el MINAM como por las regiones afectadas no fueron

Tabla 3

Presupuestos del Ministerio del Ambiente y montos destinados a conservar y restaurar el medio ambiente alterado por actividades mineras, 2011 -2016 (en soles)

\begin{tabular}{ccccc}
\hline$N^{\circ}$ & AÑO & $\begin{array}{c}\text { PRESUPUESTO MINISTERIO DEL } \\
\text { AMBIENTE PIM }\end{array}$ & $\begin{array}{c}\text { MONTO DESTINADO PARA CONSERVAR Y } \\
\text { RESTAURAR EL MEDIO AMBIENTE }\end{array}$ & \begin{tabular}{c} 
EN PORCENTAJES \\
\hline 1
\end{tabular} 2011 \\
2 & 2012 & $78,919,097$ & 0 & 0 \\
3 & 2013 & $82,816,366$ & 0 & 0 \\
4 & 2014 & $291,008.642$ & 0 & 0 \\
5 & 2015 & $235,436,138$ & 0 & 0 \\
6 & 2016 & $189,084,250$ & 0 & 0 \\
\hline & TOTAL & $230,180,605$ & 0 & 0 \\
\hline
\end{tabular}

Fuente: Adaptado de Portal de Transparencia, MINAM, 2016 (https://www.gob.pe/minam)

Tabla 4

Presupuesto Institucional Modificado (PIM) del Estado y Regiones afectadas en su medio ambiente por el sector minero y lo destinado para conservar y restaurar el medio ambiente afectado por la explotación de minería (2011 - 2016)

\begin{tabular}{|c|c|c|c|c|}
\hline $\mathbf{N}^{\circ}$ & ESTADO MINAM Y REGIONES & $\begin{array}{c}\text { PRESUPUESTOS PIM } \\
2011-2015\end{array}$ & $\begin{array}{l}\text { PRESUPUESTO } \\
\text { PIM CANON } \\
2011-2016 \\
\end{array}$ & $\begin{array}{c}\text { MONTO PARA LA CONSERVACIÓN Y } \\
\text { RESTAURACIÓN DEL MEDIO AMBIENTE } \\
2011-2016\end{array}$ \\
\hline 1 & MINISTERIO DEL AMBIENTE (MINAM) & $1,107,445,098$ & & 0 \\
\hline 2 & G.R. CAJAMARCA & $9,656,821,194$ & $1,370,639,493$ & 0 \\
\hline 3 & G.R. AMAZONAS & $3,918.110 .364$ & $202,453,489$ & 0 \\
\hline 4 & G.R. ÁNCASH & $8,849,624,329$ & $1,880,489,087$ & 0 \\
\hline 5 & G.R. PIURA & $8,861,715,864$ & $1,300,830,236$ & 0 \\
\hline 6 & G.R. HUANCAVELICA & $4,749,105,195$ & $467,481,706$ & 0 \\
\hline 7 & G.R. JUNÍN & $7,633,257,397$ & $277,814,937$ & 0 \\
\hline 8 & G.R. PASCO & $3,183,884,442$ & $444,244,391$ & 0 \\
\hline 9 & G.R. LIMA PROV. & $4,692,186,947$ & $541,532,996$ & 0 \\
\hline 10 & G.R. CALLAO & $6,320,894,615$ & $1,250,428,484$ & 0 \\
\hline 11 & G.R. PUNO & $8,255,805,186$ & $731,145,256$ & 0 \\
\hline 12 & G.R. CUSCO & $10,913,698,286$ & $3,393,292,766$ & 0 \\
\hline 13 & G.R. APURÍMAC & $4,852,768,989$ & $209,031,610$ & 0 \\
\hline 14 & G.R. AYACUCHO & $6,708.012,497$ & $500,999,437$ & 0 \\
\hline 15 & G.R. MOQUEGUA & $2,792,314.982$ & $673,692.182$ & 0 \\
\hline \multirow[t]{2}{*}{16} & G.R. TACNA & $3,210,612,590$ & $818,847,684$ & 0 \\
\hline & TOTAL & $95,706,257,975$ & $14,062,923,754$ & 0 \\
\hline
\end{tabular}

Fuente: Adaptado de Portal de Transparencia, MINAM, 2016 (https://www.gob.pe/minam) 
destinados para conservar ni restaurar el medioambiente afectado por las actividades mineras: cero a nivel porcentual.

La tabla 6 muestra la vergonzosa inacción del Estado y sus entes descentralizados, al no presentar proyecto alguno para conservar y restaurar el medioambiente dañado durante todo el periodo de estudio, aun teniendo los recursos necesarios.

Por último, la tabla 7 revela que el PIM del Estado y de las regiones afectadas en su medio ambiente por el sector minero, no destina monto alguno para la conservación y restauración del medio ambiente dañado por la explotación minera. Un panorama realmente incierto que da lugar a las conclusiones y recomendaciones de la investigación.

\section{DISCUSIÓN}

Luego del análisis de los resultados obtenidos y de establecer una comparación con el estudio de Mogrovejo (2017), quien expone la problemática de la formulación

Tabla 5

Tributación y PIM del MINAM y de las Regiones afectadas por la explotación minera (2011-2016).

\begin{tabular}{|c|c|c|c|c|c|}
\hline $\mathbf{N}^{\circ}$ & $\begin{array}{l}\text { ESTADO } \\
\text { PERUANO }\end{array}$ & $\begin{array}{l}\text { RECAUDACIÓN Y PIM } \\
\text { (En miles de millones) }\end{array}$ & $\begin{array}{c}\% \text { RECAUDACIÓN ESTADO } \\
\text { Y PIM REGIONES } \\
\text { AFECTADAS }\end{array}$ & $\begin{array}{l}\text { CONSERVACIÓN Y } \\
\text { RESTAURACIÓN DEL } \\
\text { MEDIO AMBIENTE }\end{array}$ & $\begin{array}{l}\text { \% DESTINADO A LA } \\
\text { CONSERVACIÓNY } \\
\text { RESTAURACIÓN }\end{array}$ \\
\hline 1 & $\begin{array}{l}\text { TRIBUTACIÓN ESTADO } \\
\text { PERUANO }\end{array}$ & $452,072.0$ & $100 \%$ & 0 & $0 \%$ \\
\hline 2 & $\begin{array}{l}\text { PIM DEL MINISTERIO } \\
\text { DEL AMBIENTE }\end{array}$ & $1,107.4$ & $0,24 \%$ & 0 & $0 \%$ \\
\hline 3 & $\begin{array}{l}\text { PIM REGIONES } \\
\text { AFECTADAS }\end{array}$ & $94,598.8$ & $21 \%$ & 0 & $0 \%$ \\
\hline
\end{tabular}

Fuente: Adaptado de Portal de Transparencia, MINAM, 2016 (https://www.gob.pe/minam).

Tabla 6

Proyectos presentados por el MINAM y las Regiones con medio ambiente afectado debido a explotación de minería (2011 - 2016)

\begin{tabular}{|c|c|c|c|}
\hline $\mathbf{N}^{\circ}$ & ESTADO, MINAM Y REGIONES & $\begin{array}{c}\text { PROYECTOS PRESENTADOS } \\
2011-2015\end{array}$ & $\begin{array}{l}\text { PROYECTOS PRESENTADOS PARA LA } \\
\text { CONSERVAR Y RESTAURAR EL MEDIO } \\
\text { AMBIENTE } 2011 \text {-2016 }\end{array}$ \\
\hline 1 & MINISTERIO DEL AMBIENTE (MINAM) & 5 & 0 \\
\hline 2 & G.R. CAJAMARCA & 1,611 & 0 \\
\hline 3 & G.R. AMAZONAS & 2,467 & 0 \\
\hline 4 & G.R. ÁNCASH & 2,201 & 0 \\
\hline 5 & G.R. PIURA & 1,392 & 0 \\
\hline 6 & G.R. HUANCAVELICA & 2,957 & 0 \\
\hline 7 & G.R. JUNÍN & 1,188 & 0 \\
\hline 8 & G.R. PASCO & 1,559 & 0 \\
\hline 9 & G.R. LIMA PROV & 2,173 & 0 \\
\hline 10 & G.R. CALLAO & 669 & 0 \\
\hline 11 & G.R.PUNO & 1,651 & 0 \\
\hline 12 & G.R. CUSCO & 2,214 & 0 \\
\hline 13 & G.R. APURIMAC & 1,923 & 0 \\
\hline 14 & G.R. AYACUCHO & 1,592 & 0 \\
\hline 15 & G.R. MOQUEGUA & 848 & 0 \\
\hline \multirow[t]{2}{*}{16} & G.R. TACNA & 923 & 0 \\
\hline & TOTAL & 25,368 & 0 \\
\hline
\end{tabular}

Fuente: Adaptado de Portal de Transparencia, MINAM, 2016 (https://www.gob.pe/minam) 
Tabla 7

PIM del Estado y Regiones afectadas en su medio ambiente por el sector minero y PIM para la conservación y restauración del medio ambiente dañado por la explotación minera.

\begin{tabular}{llccc}
\hline N & ESTADO MINAM Y REGIONES & $\begin{array}{c}\text { PRESUPUESTOS PIM } \\
\mathbf{2 0 1 1} \mathbf{- 2 0 1 5}\end{array}$ & $\begin{array}{c}\text { PRESUPUESTO PIM } \\
\text { CANON 2011 -2016 }\end{array}$ & $\begin{array}{c}\text { MONTO PARA RESTAURACIÓN } \\
\text { DEL MEDIO AMBIENTE } \\
\text { 2011 -2016 }\end{array}$ \\
\hline 1 & MINISTERIO DEL AMBIENTE (MINAM) & $1,107,445,098$ & & 0 \\
2 & G.R. CAJAMARCA & $9,656,821,194$ & $1,370,639,493$ & 0 \\
3 & GR AMAZONAS & $3,918.110 .364$ & $202,453,489$ & 0 \\
4 & G.R. ÁNCASH & $8,849,624,329$ & $1,880,489,087$ & 0 \\
5 & G.R. PIURA & $8,861,715,864$ & $1,300,830,236$ & 0 \\
6 & G.R. HUANCAVELICA & $4,749,105,195$ & $467,481,706$ & 0 \\
7 & G.R. JUNÍN & $7,633,257,397$ & $277,814,937$ & 0 \\
8 & G.R. PASCO & $3,183,884,442$ & $444,244,391$ & 0 \\
9 & G.R. LIMA PROV & $4,692,186,947$ & $541,532,996$ & 0 \\
10 & G.R. CALLAO & $6,320,894,615$ & $1,250,428,484$ & 0 \\
11 & G.R. PUNO & $8,255,805,186$ & $731,145,256$ & 0 \\
12 & G.R. CUSCO & $10,913,698,286$ & $3,393,292,766$ & 0 \\
13 & G.R. APURÍMAC & $4,852,768,989$ & $209,031,610$ & 0 \\
14 & G.R. AYACUCHO & $6,708.012,497$ & $500,999,437$ & 0 \\
15 & G.R. MOQUEGUA & $2,792,314.982$ & $673,692.182$ & 0 \\
16 & G.R. TACNA & $3,210,612,590$ & $818,847,684$ & 0 \\
\hline
\end{tabular}

Fuente: Adaptado de Portal de Transparencia, MINAM, 2016 (https://www.gob.pe/minam)

jurídica en el campo ambiental, revela las evidencias del principio quien contamina paga y aborda la tributación medioambiental y sus fundamentos en el país del Ecuador; se confirma la existencia real de problemas sociales, conflictos de las empresas mineras con las poblaciones, en relación con la contaminación del medio ambiente, alterando la vida de las comunidades y los ecosistemas de las regiones, por acción de la minería en el Perú. Se concluye que tanto el Ministerio del Ambiente como los gobiernos regionales afectados, no incluyen en sus PIM, en relación con sus proyectos de inversión, proyecto alguno vinculado con la conservación o restauración del ambiente. Por consiguiente, como el Estado peruano, a pesar de la tributación minera, y de los cánones mineros otorgados a las regiones, al no haber elaborado y ejecutado los proyectos de conservación y restauración del medio ambiente tanto a nivel del MINAM como de los gobiernos de las regiones de la población objetivo, no es posible comprender el daño al medio ambiente, el monto para su conservación, así como para restaurarlo, y menos aún, si la tributación del sector minero compensa el daño causado. Asimismo, el Estado peruano a través del MINAM muestra una gestión totalmente ineficaz tanto para conservar como para restaurar el ambiente afectado. El MINAM, durante el periodo de estudio, ha elaborado y ejecutado cinco proyectos de inversión pública y ninguno de ellos está destinado para conservar y restaurar el ambiente. También se evidencia una total desproporcionalidad, una asimetría absoluta, entre la recaudación de los tributos del sector minero y el gasto generado por el Estado peruano, a través del MINAM, en cuanto a acciones de restauración, así como de conservación del ambiente, especialmente de los ecosistemas afectados por la actividad minera. Este panorama se vuelve a repetir en relación a las 15 regiones de la población objetivo, así, a pesar de que estas regiones han tenido en sus PIM, durante el periodo 2011-2016, la suma de 95, 706, 257 y 975 millones de soles; sin embargo, de ese monto no se ha asignado suma alguna para acciones de conservación ni de restauración del ambiente afectado; de este modo, la desproporcionalidad es absoluta entre la tributación minera y el gasto del sector estatal con respecto a la conservación y restauración del ambiente afectado. Por último, se confirma la necesidad de una tributación destinada exclusivamente a acciones que coadyuven a conservar, así como a la restauración del ambiente y del ecosistema dañado por las actividades del sector minero. Por extensión, esta necesidad debe cubrir todos los ecosistemas afectados por otras actividades económicas, como la minería informal. 


\section{REFERENCIAS}

Asociación Interamericana para la Defensa del Ambiente. (2014). Lineamientos Básicos para la Evaluación de Impactos Ambientales de Proyectos Mineros: Términos de referencia recomendados. Recuperado de: https:// aida-americas.org/sites/default/files/publication/tdr_ mining_14-12_0.pdf.

Mariluz, O. (26 de agosto de 2014). De cada 100 supervisiones de OEFA menos de dos terminaron en multa. Gestión. Recuperado de: https:/gestion.pe/econo$\mathrm{mia} / 100$-supervisiones-oefa-dos-terminaron-multa-69160-noticia/?ref=gesr

Ministerio del Ambiente. (2016). Portal de Transparencia. Recuperado de: https://www.minam.gob.pe/transparencia-/

Mogrovejo, J. (2017). El principio de quien contamina paga y la tributación medioambiental. Una mirada del caso ecuatoriano (Tesis doctoral). Universidad Pública de Navarra, España.
Observatorio de Conflictos Mineros de América Latina. (27 de setiembre de 2018). Conflictos Mineros en Perú. Recuperado de: https://mapa.conflictosmineros.net/ ocmal_db-v2/conflicto/lista/02034800

Pizarro, P. (2016). La Tributación ambiental, un Instrumento para reducir la contaminación generada por las empresas Mineras en el Perú. Alma Mater, 3(4).

Sociedad Nacional de Minería, Petróleo y Energía. (29 de setiembre de 2018). Quiénes Somos. Asociados. Minería. Recuperado de: http://www.snmpe.org.pe/quienes-somos/asociados/mineria.html

Superintendencia Nacional de Aduanas y de Administración Tributaria. (1 de octubre de 2016). Estadísticas y Estudios. Octubre 1, 2018. Recuperado de:http://www. cva.itesm.mx/biblioteca/pagina_con_formato_version_oct/apaweb.html

Tobia, R. (2019). Una mirada comparada sobre los tributos ambientales en Chile y España. Revista de Derecho Ambiental, (12), 7-28. 